\title{
A Preliminary Study of Tinnitus Subgroups based on Self-reported Presbyacusis and Noise Induced Hearing Loss
}

\author{
Yu Wang ${ }^{1}$, Tao Pan $^{1}$, and Richard Tyler ${ }^{2}$ \\ ${ }^{1}$ Peking University Third Hospital \\ ${ }^{2}$ University of Iowa
}

November 27, 2020

\begin{abstract}
Objective: To compare some characteristics of two subgroups of tinnitus, noise-induced tinnitus and age-related tinnitus. Design: An online survey from the Iowa Tinnitus Website was reviewed. The participants were divided into two subgroups according to their reported cause of tinnitus - noise or aging. Their answers to the questions about loudness, qualities, worsening and reducing factors of tinnitus were analyzed. Study sample: There were 16 patients of age-related tinnitus and 147 of noiseinduced tinnitus. Results: Two groups did not differ significantly in loudness $(\mathrm{U}=887.5, \mathrm{p}>0.05)$, qualities $(\mathrm{X} 2=1.164$, $\mathrm{p}>$ $0.05)$ or reducing factors $(\mathrm{X} 2=1.984, \mathrm{p}>0.05)$. Ringing/whistling was the most common quality of both age-related $(37.5 \%)$ and noise-induced tinnitus (51.7\%). $42.9 \%$ of participants of noise-induced tinnitus and $31.3 \%$ of age-related tinnitus felt that nothing made tinnitus better. The main worsening factors for noise-induced tinnitus included being in a noisy place (44.2\%) and lack of sleep $(42.9 \%)$, which differed from the age-related group $(\mathrm{X} 2=6.169, \mathrm{p}<0.05)$. Conclusions: There was a great deal of overlap between two subgroups, with some differences in worsening factors, including noise environment and lack of sleep. This is an important 'red flag' in counseling, and should be considered by doctors when choosing therapy.
\end{abstract}

\section{Hosted file}

Tinnitus Subgroups Presby NIHL v25-CO format.pdf available at https://authorea.com/users/ 379462/articles/495662-a-preliminary-study-of-tinnitus-subgroups-based-on-self-reportedpresbyacusis-and-noise-induced-hearing-loss 